\title{
DECISION MAKING WHEN CHOOSING AN OPTIMAL PERSONAL BANK ACCOUNT BASED ON A MULTICRITERIAL ACCOUNT EVALUATION MODEL
}

\author{
Dorota GAWROŃSKA \\ Silesian University of Technology, Faculty of Organization and Management, Zabrze; \\ dorota.gawronska@polsl.pl, ORCID: 0000-0001-8192-0789
}

\section{Introduction}

When choosing a bank account, all available offers and the conditions offered by the banks should be analysed. People who already have accounts rarely change the bank. However, sometimes unsatisfactory issues happen in cooperation with a given bank, which somewhat "forces" the customer to take steps to start the process of searching for a new option. Banks not only advertise terms for their customers but also try to outdo each other in offers designed to attract new customers: whether they have resigned from cooperation with another bank or they open a personal account for the first time, e.g. as a result of their first job. It seems reasonable to create a model that helps in selection of a new account that best reflects the expectations of a potential new customer.

The purpose of the study is to make a comparative analysis of the 12 most popular personal accounts proposed by banks. The criteria are the most important points which potential clients take into consideration:

1. Initial fee when opening an account.

2. Account maintenance fee:

- free,

- free with the condition of making a certain number of transactions,

- free with the condition of making a transaction for a specific amount,

- free with the condition of making a deposit of a certain amount in the previous month,

- free for customers under a certain age. 
3. Card fee:

- free,

- free with the condition of making a certain number of transactions,

- free with the condition of making a transaction for a specific amount,

- free with the condition of making a deposit of a certain amount in the previous month,

- free for customers under a certain age.

4. Fees for using cash machines:

- all cash machines free of charge,

- fees for using other banks' cash machines,

- fees for using banks' services abroad.

5. Additional options:

- Apple Pay,

- Google Pay,

- Blik

- Cashback.

6. Account opening bonus.

7. Special interest rates on deposits.

Below, tables (1-3) present offers of 12 banks regarding personal bank accounts for new customers.

\section{Multicriterial analysis model}

Coping with the search for an optimal solution, when dealing with multiple criteria, various methods can be used (Trzaskalik, 2006, Stadnicki, 2006). This paper presents one of the possibilities of solving the problem based on the hierarchical structure of criteria, taking into account the importance of criteria.

In the presented model, 12 bank accounts belonging to the finite set B are analysed:

$B=\left\{B_{1}, B_{2}, \ldots, B_{i}, \ldots, B_{12}\right\}, \quad i=1, \ldots, 12$,

The criteria presented in point 1 of the paper form a two-level hierarchical structure.

The variable $\mathrm{Vl}$ is adopted in the paper, determining the importance of assessments against Level 1 criteria:

$\sum_{l=1}^{7} V_{l}=1$

The importance of Level 2 criteria is described by the variable VLM ( $\mathrm{m}$ - Level 2 criterion).

$\sum_{m} V_{l m}=1 .$. 
Table 1.

Statement of fees related to the opening and maintenance of the account and fees related to the card

\begin{tabular}{|c|c|c|c|c|c|c|c|c|}
\hline & $\begin{array}{c}\text { Account } \\
\text { opening fee }\end{array}$ & $\begin{array}{c}\text { Account } \\
\text { maintenance fee } \\
\text { if conditions are } \\
\text { not met }\end{array}$ & $\begin{array}{l}\text { Free account } \\
\text { maintenance with a } \\
\text { certain number of } \\
\text { transactions }\end{array}$ & $\begin{array}{c}\text { Free account } \\
\text { maintenance } \\
\text { with a specific } \\
\text { deposit made } \\
\text { into the account }\end{array}$ & $\begin{array}{c}\text { Free account } \\
\text { maintenance for } \\
\text { customers under a } \\
\text { certain age }\end{array}$ & $\begin{array}{l}\text { Card fee if } \\
\text { conditions } \\
\text { are not met }\end{array}$ & $\begin{array}{c}\text { Required } \\
\text { transaction } \\
\text { amount for a } \\
\text { free card }\end{array}$ & $\begin{array}{l}\text { Number of } \\
\text { transactions } \\
\text { required for a } \\
\text { free card }\end{array}$ \\
\hline $\begin{array}{c}\text { Konto Direct - } \\
\text { ING Bank Śląski } \\
\text { eKonto standard - } \\
\text { mBank }\end{array}$ & 0 & 0 & 0 & 0 & 0 & 7 & 300 & 0 \\
\hline $\begin{array}{c}\text { eKonto standard - } \\
\text { mBank }\end{array}$ & 0 & 0 & 5 & 0 & 0 & 4 & 0 & 0 \\
\hline $\begin{array}{l}\text { Nest Account - } \\
\text { Nest Bank }\end{array}$ & 0 & 0 & 0 & 0 & 0 & 0 & 0 & 0 \\
\hline $\begin{array}{c}\text { Konto Proste } \\
\text { Zasady - Getin } \\
\text { Bank } \\
\end{array}$ & 0 & 8 & 1 & 0 & 0 & 0 & 0 & 0 \\
\hline $\begin{array}{l}\text { Konto Optymalne- } \\
\text { PNB Paribas }\end{array}$ & 0 & 0 & 0 & 0 & 0 & 0 & 0 & 0 \\
\hline Millenium & 0 & 8 & 1 & 1,000 & 0 & 0 & 0 & 0 \\
\hline $\begin{array}{c}\text { Konto dla Ciebie - } \\
\text { Credit Agricole }\end{array}$ & 0 & 0 & 0 & 0 & 0 & 0 & 0 & 0 \\
\hline $\begin{array}{c}\text { Konto na Zero - } \\
\text { PKO BP }\end{array}$ & 0 & 6.9 & 0 & 0 & 0 & 6.9 & 300 & 0 \\
\hline $\begin{array}{c}\text { Konto Jakże } \\
\text { Osobiste - Alior } \\
\text { Bank } \\
\end{array}$ & 0 & 10 & 0 & 1,500 & 26 & 10 & 300 & 0 \\
\hline $\begin{array}{c}\text { Konto Freemium - } \\
\text { T-mobile Usługi } \\
\text { Bankowe }\end{array}$ & 0 & 0 & 0 & 0 & 0 & 6 & 200 & 0 \\
\hline $\begin{array}{c}\text { Konto Jakie Chcę- } \\
\text { Santander Bank } \\
\text { Polska }\end{array}$ & 0 & 0 & 0 & 0 & 0 & 5 & 0 & 5 \\
\hline $\begin{array}{c}\text { Konto Bez Granic } \\
\text { - PKO BP }\end{array}$ & 17.9 & 0 & 0 & 0 & 0 & 0 & 0 & 0 \\
\hline
\end{tabular}

Note: own study based on: https://www.rankingkontosobistych.pl/konta-osobiste. 
Table 2.

Statement of costs of withdrawals from cash machines of different banks or cash machines abroad

\begin{tabular}{|c|c|c|c|c|c|c|c|c|c|c|c|}
\hline & $\begin{array}{c}\text { The cost of } \\
\text { withdrawing } \\
\text { from cash } \\
\text { machines of } \\
\text { different banks } \\
\text { when the } \\
\text { conditions are } \\
\text { not met } \\
\end{array}$ & \begin{tabular}{|c|} 
Free \\
withdrawal \\
from cash \\
machines of \\
different \\
banks upon \\
authorization \\
by Blik \\
\end{tabular} & $\begin{array}{c}\text { Free } \\
\text { withdrawal } \\
\text { when a } \\
\text { certain } \\
\text { deposit was } \\
\text { made }\end{array}$ & $\begin{array}{c}\text { Number of } \\
\text { transactions } \\
\text { for free } \\
\text { withdrawal }\end{array}$ & $\begin{array}{l}\text { Withdrawal } \\
\text { from cash } \\
\text { machines of } \\
\text { different } \\
\text { banks\% }\end{array}$ & $\begin{array}{c}\begin{array}{c}\text { Number of } \\
\text { free }\end{array} \\
\text { withdrawals } \\
\text { from cash } \\
\text { machines of } \\
\text { different } \\
\text { banks }\end{array}$ & $\begin{array}{l}\text { The cost of } \\
\text { withdrawals } \\
\text { from cash } \\
\text { machines } \\
\text { abroad when } \\
\text { the conditions } \\
\text { are not met }\end{array}$ & $\begin{array}{c}\text { The cost of } \\
\text { withdrawals } \\
\text { from cash } \\
\text { machines } \\
\text { abroad \% }\end{array}$ & $\begin{array}{c}\text { Free } \\
\text { withdrawal } \\
\text { on payment } \\
\text { to the } \\
\text { account of a } \\
\text { certain } \\
\text { amount }\end{array}$ & $\begin{array}{c}\text { Number of } \\
\text { transactions } \\
\text { for free } \\
\text { withdrawal }\end{array}$ & $\begin{array}{c}\begin{array}{c}\text { Number of } \\
\text { free }\end{array} \\
\text { withdrawals } \\
\text { abroad }\end{array}$ \\
\hline $\begin{array}{c}\text { Konto Direct } \\
\text { - ING Bank } \\
\text { Śląski }\end{array}$ & 2.5 & 0 & 0 & 0 & 0 & 0 & 0 & $3 \%$ & 0 & 0 & 1 \\
\hline $\begin{array}{l}\text { eKonto } \\
\text { standard - } \\
\text { mBank }\end{array}$ & 7 & 0 & 0 & 0 & 0 & 0 & $10 \mathrm{zl}$ & $5.90 \%$ & 0 & 0 & 0 \\
\hline $\begin{array}{l}\text { Nest Account } \\
\text { - Nest Bank }\end{array}$ & 0 & 0 & 0 & 0 & 0 & 0 & $5 \mathrm{zl}$ & $3 \%$ & 0 & 0 & 0 \\
\hline $\begin{array}{l}\text { Konto Proste } \\
\text { Zasady - } \\
\text { Getin Bank }\end{array}$ & 0 & 0 & 0 & 0 & 0 & 0 & $10 \mathrm{zl}$ & $4.50 \%$ & 0 & 0 & 0 \\
\hline $\begin{array}{c}\text { Konto } \\
\text { Optymalne- } \\
\text { PNB Paribas }\end{array}$ & 5 & 0 & 0 & 0 & 0 & 0 & $10 \mathrm{zl}$ & 0 & 0 & 0 & 0 \\
\hline Millenium & 5 & 0 & 1,000 & 1 & 0 & 0 & 9 & $2.50 \%$ & 1,000 & 1 & 0 \\
\hline $\begin{array}{c}\text { Konto Dla } \\
\text { Ciebie - } \\
\text { Credit } \\
\text { Agricole }\end{array}$ & 5 & 0 & 1,000 & 1 & 0 & 0 & 10 & 0 & 0 & 0 & 0 \\
\hline $\begin{array}{c}\text { Konto } \mathrm{Na} \\
\text { zero - } \mathrm{PKO} \\
\mathrm{BP}\end{array}$ & 5 & 0 & 0 & 0 & $3 \%$ & 0 & 10 & $3 \%$ & 0 & 0 & 0 \\
\hline $\begin{array}{c}\text { Konto Jakże } \\
\text { Osobiste - } \\
\text { Alior Bank }\end{array}$ & 2.50 & 0 & 0 & 0 & 0 & 0 & $5 \mathrm{zl}$ & 0 & 0 & 0 & 0 \\
\hline
\end{tabular}


Cont. table 2 .

\begin{tabular}{|c|c|c|c|c|c|c|c|c|c|c|}
\hline $\begin{array}{c}\text { Konto } \\
\text { Freemium - } \\
\text { T-mobile } \\
\text { ustugi } \\
\text { Bankowe }\end{array}$ & 6 & 0 & 0 & 0 & 0 & 3 & 6 & 0 & 0 & 3 \\
\hline $\begin{array}{c}\text { Konto Jakie } \\
\text { Chce - } \\
\begin{array}{c}\text { Santander } \\
\text { Bank Polska }\end{array}\end{array}$ & 5 & 1 & 0 & 0 & 0 & 0 & 10 & $2.50 \%$ & 0 & 0 \\
\hline $\begin{array}{c}\text { Konto Bez } \\
\text { Granic - PKO } \\
\text { BP }\end{array}$ & 0 & 0 & 0 & 0 & 0 & 0 & 0 & 0 & 0 & 0 \\
\hline
\end{tabular}

Note: own study based on: https://www.rankingkontosobistych.pl/konta-osobiste. 
Table 3.

Options availability statement

\begin{tabular}{|c|c|c|c|c|c|c|}
\hline & $\begin{array}{l}\text { Apple } \\
\text { Pay }\end{array}$ & $\begin{array}{c}\text { Google } \\
\text { Pay }\end{array}$ & Blik & Cashback & $\begin{array}{c}\text { Maximum bonus for } \\
\text { setting up an account } \\
{[\mathrm{zl}]}\end{array}$ & $\begin{array}{c}\text { Special deposit } \\
\%\end{array}$ \\
\hline $\begin{array}{c}\text { Konto Direct - ING } \\
\text { Bank Śląski } \\
\end{array}$ & Yes & Yes & Yes & No & 140 & 2.5 \\
\hline $\begin{array}{c}\text { eKonto standard - } \\
\text { mBank }\end{array}$ & Yes & Yes & Yes & Yes & 140 & 0 \\
\hline $\begin{array}{c}\text { Nest Account - Nest } \\
\text { Bank }\end{array}$ & Yes & Yes & No & No & 0 & 4 \\
\hline $\begin{array}{c}\text { Konto Proste Zasady } \\
\text { - Getin Bank }\end{array}$ & Yes & Yes & Yes & Yes & 0 & 3.5 \\
\hline $\begin{array}{c}\text { Konto Optymalne - } \\
\text { PNB Paribas }\end{array}$ & Yes & Yes & Yes & Yes & 300 & 0 \\
\hline Millenium & Yes & No & Yes & No & 0 & 2.7 \\
\hline $\begin{array}{l}\text { Konto Dla Ciebie - } \\
\text { Credit Agricole }\end{array}$ & Yes & Yes & Yes & Yes & 300 & 0 \\
\hline $\begin{array}{c}\text { Konto Na Zero - } \\
\text { PKO BP }\end{array}$ & Yes & No & Yes & Yes & 0 & 0 \\
\hline $\begin{array}{c}\text { Konto Jakże Osobiste } \\
\text { - Alior Bank }\end{array}$ & Yes & Yes & Yes & Yes & 0 & 0 \\
\hline $\begin{array}{c}\text { Konto Freemium - } \\
\text { T-mobile Usługi } \\
\text { Bankowe }\end{array}$ & Yes & Yes & Yes & No & 0 & 0 \\
\hline $\begin{array}{c}\text { Konto Jakie Chcę- } \\
\text { Santander Bank } \\
\text { Polska } \\
\end{array}$ & Yes & Yes & Yes & Yes & 0 & 0 \\
\hline $\begin{array}{c}\text { Konto Bez Granic - } \\
\text { PKO BP }\end{array}$ & Yes & Yes & Yes & Yes & 0 & 0 \\
\hline
\end{tabular}

Note: own study based on https://www.rankingkontosobistych.pl/konta-osobiste.

At the beginning of the assessment process, we standardise assessments by the following formula:

a) for the criteria against which the optimisation comes down to searching for the maximum value:

$K_{i l m}^{,}=\frac{K_{i l m}}{\max K_{i l m}}$,

b) for the criteria against which the optimisation comes down to the search for a minimum value:

$$
K_{i l m}^{,}=1-\frac{K_{i l m}}{\max K_{i l m}} .
$$

Based on standardised assessments, the first stage of the analysis can begin, i.e., specifying cumulative assessments against Level 2 criteria:

$$
K_{i l}^{\prime}=\frac{\sum_{m} V_{m} \cdot K_{i l m}^{\prime}}{\sum_{m} V_{m}} .
$$


After determining the level 2 assessments, we normalise again according to the following formulas:

a) for criteria: additional options, bonus for opening an account, promotional interest on deposits:

$$
K_{i l}=\frac{K_{i l}^{\prime}}{\max K_{i l}^{\prime}},
$$

b) for the criteria: initial payment when opening an account, account maintenance fee, card fee, fees for using cash machines:

$$
K_{i l}=1-\frac{K_{i l}^{\prime}}{\max K_{i l}^{\prime}} .
$$

After normalisation, the next stage of the analysis is to determine cumulative assessments against Level 1 criteria:

$$
K_{i}^{\prime}=\frac{\sum_{l} V_{l} \cdot K_{i l}^{\prime}}{\sum_{l} V_{l}} .
$$

Due to the adopted normalisation method (formulas (4)-(5) and (7)-(8)), the model strives for the maximum assessment, which indicates the bank account best suited to the needs:

$K_{i} \rightarrow M A X$

\section{The results of the analysis of bank accounts}

Based on the presented in the study in point 2 work of the model for multicriterial assessment of bank accounts, the proposed offers were evaluated. The calculations assume the same level of criteria importance, but the model assumes the possibility of determining different weights for individual criteria. The reason why the equal weights were adopted is that depending on the expectations of potential customers, the weights would have different values. And although creating a pattern of behaviour of groups of new customers could be tempting, this is not the subject of this work.

According to the algorithm, the first step is the normalisation of assessments. They are presented in the tables 4-6. 
Table 4.

Normalised assessment values for account opening, account maintenance and card

\begin{tabular}{|c|c|c|c|c|c|c|c|c|}
\hline & $\begin{array}{c}\text { Fee for } \\
\text { opening } \\
\text { an } \\
\text { account }\end{array}$ & $\begin{array}{c}\text { Account } \\
\text { maintenance } \\
\text { fee if } \\
\text { conditions } \\
\text { are not met }\end{array}$ & $\begin{array}{c}\text { Free } \\
\text { account } \\
\text { maintenance } \\
\text { with a } \\
\text { certain } \\
\text { number of } \\
\text { transactions } \\
\end{array}$ & $\begin{array}{l}\text { Free account } \\
\text { maintenance } \\
\text { with a } \\
\text { specific } \\
\text { deposit made } \\
\text { into the } \\
\text { account }\end{array}$ & $\begin{array}{c}\text { Free account } \\
\text { maintenance } \\
\text { for customers } \\
\text { under a } \\
\text { certain age }\end{array}$ & $\begin{array}{l}\text { Card fee if } \\
\text { conditions } \\
\text { are not met }\end{array}$ & $\begin{array}{c}\text { Required } \\
\text { transaction } \\
\text { amount for a } \\
\text { free card }\end{array}$ & $\begin{array}{l}\text { Number of } \\
\text { transactions } \\
\text { required } \\
\text { for a free } \\
\text { card }\end{array}$ \\
\hline $\begin{array}{c}\text { KontoDirect } \\
\text { - ING Bank } \\
\text { Śląski }\end{array}$ & 1.00 & 1.00 & 1.00 & 1.00 & 1.00 & 0.30 & 0.00 & 1.00 \\
\hline $\begin{array}{c}\text { eKonto } \\
\text { standard - } \\
\text { mBank } \\
\end{array}$ & 1.00 & 1.00 & 0.00 & 1.00 & 1.00 & 0.60 & 1.00 & 1.00 \\
\hline $\begin{array}{c}\text { Nest } \\
\text { Account - } \\
\text { Nest Bank }\end{array}$ & 1.00 & 1.00 & 1.00 & 1.00 & 1.00 & 1.00 & 1.00 & 1.00 \\
\hline $\begin{array}{c}\text { Konto } \\
\text { Proste } \\
\text { Zasady - } \\
\text { Getin Bank }\end{array}$ & 1.00 & 0.20 & 0.80 & 1.00 & 1.00 & 1.00 & 1.00 & 1.00 \\
\hline $\begin{array}{c}\text { Konto } \\
\text { Optymalne } \\
\text { - PNB } \\
\text { Paribas }\end{array}$ & 1.00 & 1.00 & 1.00 & 1.00 & 1.00 & 1.00 & 1.00 & 1.00 \\
\hline Millenium & 1.00 & 0.20 & 0.80 & 0.33 & 1.00 & 1.00 & 1.00 & 1.00 \\
\hline $\begin{array}{c}\text { Konto Dla } \\
\text { Ciebie - } \\
\text { Credit } \\
\text { Agricole } \\
\end{array}$ & 1.00 & 1.00 & 1.00 & 1.00 & 1.00 & 1.00 & 1.00 & 1.00 \\
\hline $\begin{array}{c}\text { Konto } \mathrm{Na} \\
\text { Zero - PKO } \\
\text { BP }\end{array}$ & 1.00 & 0.31 & 1.00 & 1.00 & 1.00 & 0.31 & 0.00 & 1.00 \\
\hline $\begin{array}{c}\text { Konto Jakże } \\
\text { Osobiste - } \\
\text { Alior Bank } \\
\end{array}$ & 1.00 & 0.00 & 1.00 & 0.00 & 0.00 & 0.00 & 0.00 & 1.00 \\
\hline $\begin{array}{c}\text { Konto } \\
\text { Freemium - } \\
\text { T-mobile } \\
\text { Usługi } \\
\text { Bankowe } \\
\end{array}$ & 1.00 & 1.00 & 1.00 & 1.00 & 1.00 & 0.40 & 0.33 & 1.00 \\
\hline $\begin{array}{c}\text { Konto Jakie } \\
\text { Chcę - } \\
\text { Santander } \\
\text { Bank Polska }\end{array}$ & 1.00 & 1.00 & 1.00 & 1.00 & 1.00 & 0.50 & 1.00 & 0.00 \\
\hline $\begin{array}{c}\text { Konto Bez } \\
\text { Granic - } \\
\text { PKO BP }\end{array}$ & 0.00 & 1.00 & 1.00 & 1.00 & 1.00 & 1.00 & 1.00 & 1.00 \\
\hline
\end{tabular}

Note: own study. 
Table 5.

Normalised cost values for cash machines withdrawals

\begin{tabular}{|c|c|c|c|c|c|c|c|c|c|c|c|}
\hline & 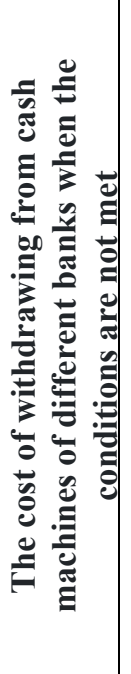 & 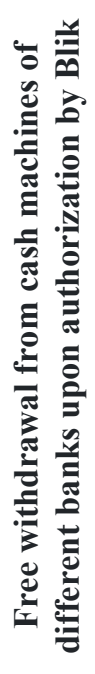 & 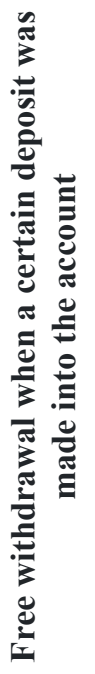 & 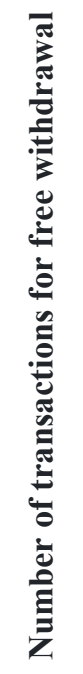 & 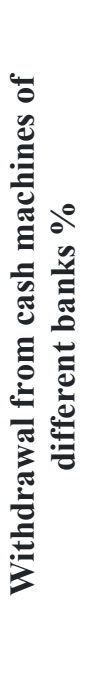 & 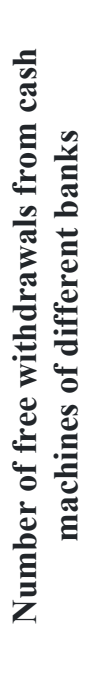 & 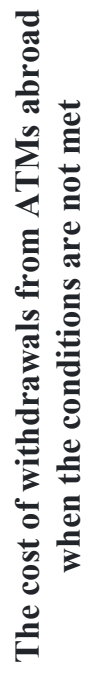 & 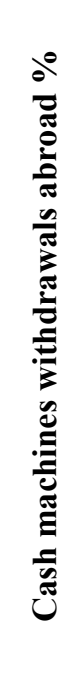 & 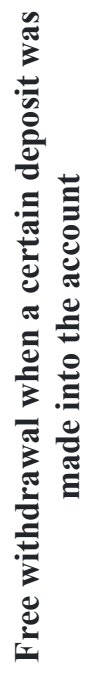 & 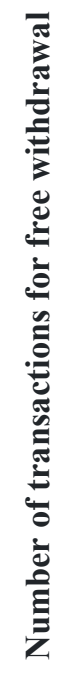 & 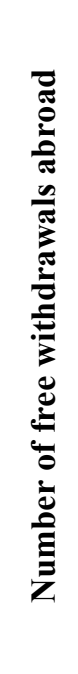 \\
\hline $\begin{array}{c}\text { Konto } \\
\text { Direct - } \\
\text { ING Bank } \\
\text { Śląski } \\
\end{array}$ & 0.64 & 1.00 & 1.00 & 1.00 & 1.00 & 1.00 & 1.00 & 0.49 & 1.00 & 1.00 & 0.67 \\
\hline $\begin{array}{c}\text { eKonto } \\
\text { standard - } \\
\text { mBank }\end{array}$ & 0.00 & 1.00 & 1.00 & 1.00 & 1.00 & 1.00 & 0.00 & 0.00 & 1.00 & 1.00 & 1.00 \\
\hline $\begin{array}{c}\text { Nest } \\
\text { Account - } \\
\text { Nest Bank }\end{array}$ & 1.00 & 1.00 & 1.00 & 1.00 & 1.00 & 1.00 & 0.50 & 0.49 & 1.00 & 1.00 & 1.00 \\
\hline $\begin{array}{c}\text { Konto } \\
\text { Proste } \\
\text { Zasady - } \\
\text { Getin } \\
\text { Bank } \\
\end{array}$ & 1.00 & 1.00 & 1.00 & 1.00 & 1.00 & 1.00 & 0.00 & 0.24 & 1.00 & 1.00 & 1.00 \\
\hline $\begin{array}{c}\text { Konto } \\
\text { Optymalne } \\
\text { - PNB } \\
\text { Paribas }\end{array}$ & 0.29 & 1.00 & 1.00 & 1.00 & 1.00 & 1.00 & 0.00 & 1.00 & 1.00 & 1.00 & 1.00 \\
\hline Millenium & 0.29 & 1.00 & 0.00 & 0.00 & 1.00 & 1.00 & 0.10 & 0.58 & 0.00 & 0.67 & 1.00 \\
\hline $\begin{array}{c}\text { Konto Dla } \\
\text { Ciebie - } \\
\text { Credit } \\
\text { Agricole } \\
\end{array}$ & 0.29 & 1.00 & 0.00 & 0.00 & 1.00 & 1.00 & 0.00 & 1.00 & 1.00 & 1.00 & 1.00 \\
\hline $\begin{array}{c}\text { Konto Na } \\
\text { Zero - } \\
\text { PKO BP }\end{array}$ & 0.29 & 1.00 & 1.00 & 1.00 & 0.00 & 1.00 & 0.00 & 0.49 & 1.00 & 1.00 & 1.00 \\
\hline $\begin{array}{c}\text { Konto } \\
\text { Jakże } \\
\text { Osobiste }- \\
\text { Alior } \\
\text { Bank }\end{array}$ & 0.64 & 1.00 & 1.00 & 1.00 & 1.00 & 1.00 & 0.50 & 1.00 & 1.00 & 1.00 & 1.00 \\
\hline $\begin{array}{c}\text { Konto } \\
\text { Freemium } \\
- \\
\text { T-mobile } \\
\text { Usługi } \\
\text { Bankowe } \\
\end{array}$ & 0.14 & 1.00 & 1.00 & 1.00 & 1.00 & 0.00 & 0.40 & 1.00 & 1.00 & 0.00 & 0.00 \\
\hline
\end{tabular}


Cont. table 5.

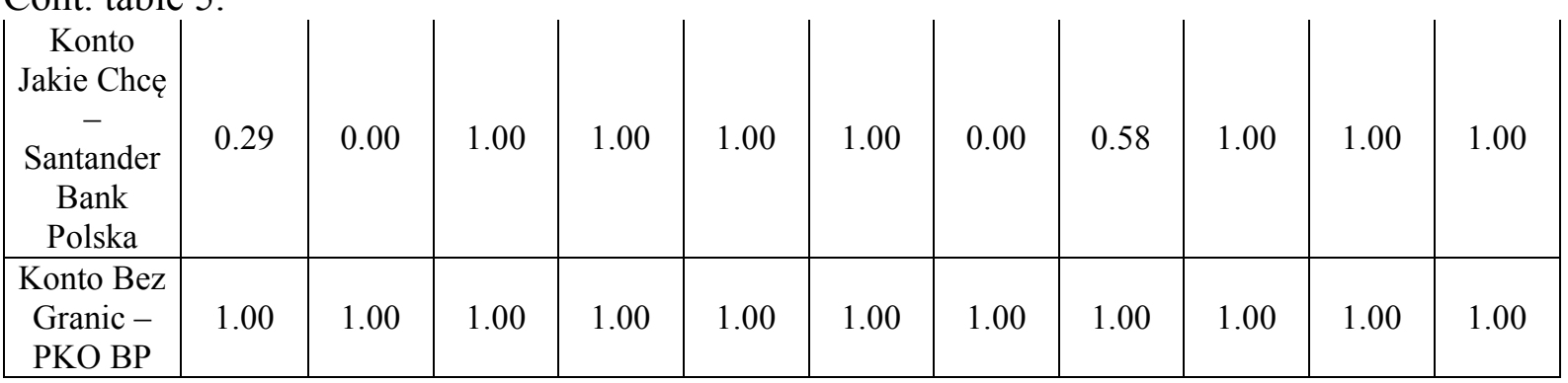

Note: own study.

Table 6.

Normalised assessment values for additional options, account opening bonuses and promotional deposits

\begin{tabular}{|c|c|c|c|c|c|c|}
\hline & Apple Pay & Google Pay & Blik & Cashback & $\begin{array}{c}\text { Maximum bonus for } \\
\text { setting up an account }\end{array}$ & $\begin{array}{c}\text { Promotional } \\
\text { deposit \% }\end{array}$ \\
\hline $\begin{array}{c}\text { Konto Direct - ING } \\
\text { Bank Sląski }\end{array}$ & 1.00 & 1.00 & 1.00 & 0.00 & 0.47 & 0.63 \\
\hline $\begin{array}{c}\text { eKonto standard - } \\
\text { mBank }\end{array}$ & 1.00 & 1.00 & 1.00 & 1.00 & 0.47 & 0.00 \\
\hline $\begin{array}{c}\text { Nest Account - Nest } \\
\text { Bank }\end{array}$ & 1.00 & 1.00 & 0.00 & 0.00 & 0.00 & 1.00 \\
\hline $\begin{array}{c}\text { Konto Proste Zasady } \\
\text { - Getin Bank }\end{array}$ & 1.00 & 1.00 & 1.00 & 1.00 & 0.00 & 0.88 \\
\hline $\begin{array}{c}\text { Konto Optymalne - } \\
\text { PNB Paribas }\end{array}$ & 1.00 & 1.00 & 1.00 & 1.00 & 1.00 & 0.00 \\
\hline $\begin{array}{c}\text { Millenium } \\
\text { Konto Dla Ciebie - } \\
\text { Credit Agricole }\end{array}$ & 1.00 & 0.00 & 1.00 & 0.00 & 0.00 & 0.68 \\
\hline $\begin{array}{c}\text { Konto Na Zero - } \\
\text { PKO BP }\end{array}$ & 1.00 & 0.00 & 1.00 & 1.00 & 0.00 & 0.00 \\
\hline $\begin{array}{c}\text { Konto Jakże Osobiste } \\
- \text { Alior Bank }\end{array}$ & 1.00 & 1.00 & 1.00 & 1.00 & 0.00 & 0.00 \\
\hline $\begin{array}{c}\text { Konto Freemium - } \\
\text { T-mobile Banking } \\
\text { services }\end{array}$ & 1.00 & 1.00 & 1.00 & 0.00 & 0.00 & 0.00 \\
\hline $\begin{array}{c}\text { Konto Jakie Chce - } \\
\text { Santander Bank } \\
\text { Polska }\end{array}$ & 1.00 & 1.00 & 1.00 & 1.00 & 0.00 & 0.00 \\
\hline $\begin{array}{c}\text { Konto Bez Granic - } \\
\text { PKO BP }\end{array}$ & 1.00 & 0.00 & 1.00 & 1.00 & 0.00 & 0.00 \\
\hline
\end{tabular}

Note: own study.

The table below presents the values of the weights of individual criteria.

Table 7.

Values of accepted weights

\begin{tabular}{|c|c|c|c|c|c|c|c|}
\hline Weight & $\begin{array}{c}\text { Weight } \\
\text { value }\end{array}$ & Weight & $\begin{array}{c}\text { Weight } \\
\text { value }\end{array}$ & Weight & $\begin{array}{c}\text { Weight } \\
\text { value }\end{array}$ & Weight & $\begin{array}{c}\text { Weight } \\
\text { value }\end{array}$ \\
\hline $\mathrm{V}_{1}$ & 0.14 & $\mathrm{~V}_{4}$ & 0.14 & $\mathrm{~V}_{5}$ & 0.14 & $\mathrm{~V}_{6}$ & 0.14 \\
\hline $\mathrm{V}_{2}$ & 0.14 & $\mathrm{~V}_{41}$ & 0.17 & $\mathrm{~V}_{51}$ & 0.2 & $\mathrm{~V}_{61}$ & 0.25 \\
\hline $\mathrm{V}_{21}$ & 0.25 & $\mathrm{~V}_{41}$ & 0.17 & $\mathrm{~V}_{52}$ & 0.2 & $\mathrm{~V}_{62}$ & 0.25 \\
\hline $\mathrm{V}_{22}$ & 0.25 & $\mathrm{~V}_{43}$ & 0.17 & $\mathrm{~V}_{53}$ & 0.2 & $\mathrm{~V}_{63}$ & 0.25 \\
\hline $\mathrm{V}_{23}$ & 0.25 & $\mathrm{~V}_{44}$ & 0.17 & $\mathrm{~V}_{54}$ & 0.2 & $\mathrm{~V}_{64}$ & 0.25 \\
\hline $\mathrm{V}_{24}$ & 0.25 & $\mathrm{~V}_{45}$ & 0.17 & $\mathrm{~V}_{55}$ & 0.2 & $\mathrm{~V}_{7}$ & 0.14 \\
\hline
\end{tabular}


Cont. table 7.

\begin{tabular}{|c|c|c|c|c|c|c|c|}
$\mathrm{V}_{3}$ & 0.14 & $\mathrm{~V}_{46}$ & 0.17 & & & & \\
\hline $\mathrm{V}_{31}$ & 0.33 & & & & & & \\
\hline $\mathrm{V}_{32}$ & 0.33 & & & & & & \\
\hline $\mathrm{V}_{33}$ & 0.33 & & & & & & \\
\hline
\end{tabular}

Note: own study.

Based on the total assessment against Level 2 criteria, total assessment of bank accounts was obtained, broken down into Level 1 criteria - the results are presented in Table 8.

Table 8.

Weighted assessments against Level 1 criteria

\begin{tabular}{|c|c|c|c|c|c|c|c|c|}
\hline & 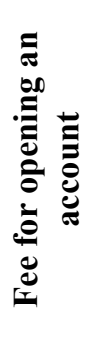 & 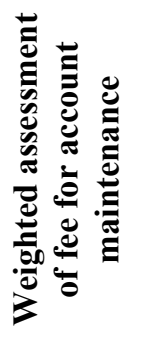 & 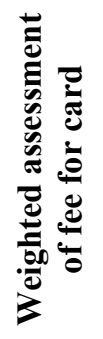 & 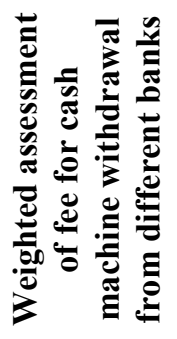 & 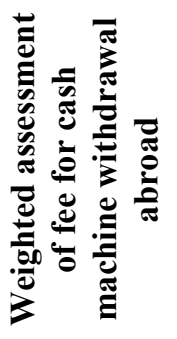 & 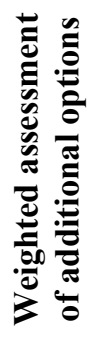 & 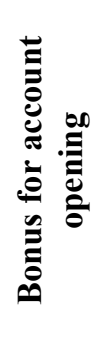 & 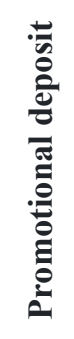 \\
\hline $\begin{array}{c}\text { Konto } \\
\text { Direct - } \\
\text { ING Bank } \\
\text { Śląski }\end{array}$ & 1.00 & 1.00 & 0.43 & 0.94 & 0.83 & 0.75 & 0.47 & 0.63 \\
\hline $\begin{array}{l}\text { eKonto } \\
\text { standard - } \\
\text { mBank }\end{array}$ & 1.00 & 0.75 & 0.87 & 0.83 & 0.60 & 1.00 & 0.47 & 0.00 \\
\hline $\begin{array}{c}\text { Nest } \\
\text { Account - } \\
\text { Nest Bank }\end{array}$ & 1.00 & 1.00 & 1.00 & 1.00 & 0.80 & 0.50 & 0.00 & 1.00 \\
\hline $\begin{array}{c}\text { Konto } \\
\text { Proste } \\
\text { Zasady - } \\
\text { Getin Bank }\end{array}$ & 1.00 & 0.75 & 1.00 & 1.00 & 0.65 & 1.00 & 0.00 & 0.88 \\
\hline $\begin{array}{c}\text { Konto } \\
\text { Optymalne } \\
\text { - PNB } \\
\text { Paribas }\end{array}$ & 1.00 & 1.00 & 1.00 & 0.88 & 0.80 & 1.00 & 1.00 & 0.00 \\
\hline Millenium & 1.00 & 0.75 & 1.00 & 0.55 & 0.47 & 0.50 & 0.00 & 0.68 \\
\hline $\begin{array}{c}\text { Konto Dla } \\
\text { Ciebie - } \\
\text { Credit } \\
\text { Agricole }\end{array}$ & 1.00 & 1.00 & 1.00 & 0.55 & 0.80 & 1.00 & 1.00 & 0.00 \\
\hline $\begin{array}{c}\text { Konto } \mathrm{Na} \\
\text { Zero - PKO } \\
\text { BP }\end{array}$ & 1.00 & 0.83 & 0.44 & 0.71 & 0.70 & 0.75 & 0.00 & 0.00 \\
\hline $\begin{array}{c}\text { Konto } \\
\text { Jakże } \\
\text { Osobiste - } \\
\text { Alior Bank }\end{array}$ & 1.00 & 0.75 & 0.33 & 0.94 & 0.90 & 1.00 & 0.00 & 0.00 \\
\hline $\begin{array}{c}\text { Konto } \\
\text { Freemium - } \\
\text { T-mobile } \\
\text { Usługi } \\
\text { Bankowe }\end{array}$ & 1.00 & 1.00 & 0.58 & 0.69 & 0.48 & 0.75 & 0.00 & 0.00 \\
\hline
\end{tabular}


Cont. table 8 .

\begin{tabular}{|c|c|c|c|c|c|c|c|c|}
\hline $\begin{array}{c}\text { Konto Jakie } \\
\text { Chce - } \\
\text { Santander } \\
\text { Bank } \\
\text { Polska }\end{array}$ & 1.00 & 1.00 & 0.50 & 0.71 & 0.72 & 1.00 & 0.00 & 0.00 \\
\hline $\begin{array}{c}\text { Konto Bez } \\
\text { Granic - } \\
\text { PKO BP }\end{array}$ & 0.00 & 1.00 & 1.00 & 1.00 & 1.00 & 0.75 & 0.00 & 0.00 \\
\hline
\end{tabular}

Note: own study.

The next step is to normalise the cumulative assessment against Level 1 criteria, as shown in Table 9.

Table 9.

Standardised assessments against Level 2 criteria

\begin{tabular}{|c|c|c|c|c|c|c|c|c|}
\hline & 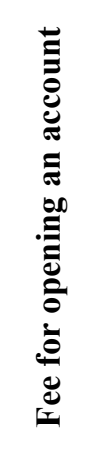 & 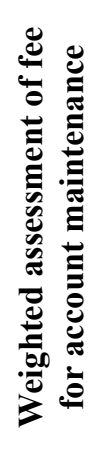 & 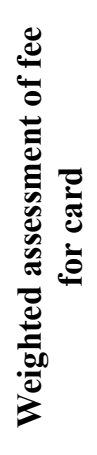 & 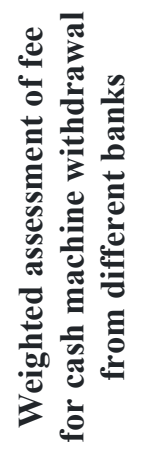 & 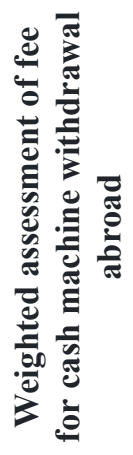 & 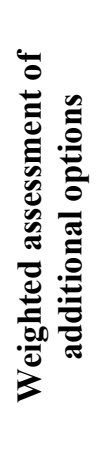 & 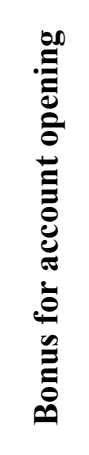 & 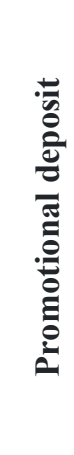 \\
\hline $\begin{array}{c}\text { Konto Direct } \\
\text { - ING Bank } \\
\text { Śląski } \\
\end{array}$ & 1.00 & 1.00 & 0.43 & 0.94 & 0.83 & 0.75 & 0.47 & 0.63 \\
\hline $\begin{array}{c}\text { eKonto } \\
\text { standard - } \\
\text { mBank }\end{array}$ & 1.00 & 0.75 & 0.87 & 0.83 & 0.60 & 1.00 & 0.47 & 0.00 \\
\hline $\begin{array}{l}\text { Nest Account } \\
\text { - Nest Bank }\end{array}$ & 1.00 & 1.00 & 1.00 & 1.00 & 0.80 & 0.50 & 0.00 & 1.00 \\
\hline $\begin{array}{c}\text { Konto Proste } \\
\text { Zasady - } \\
\text { Getin Bank }\end{array}$ & 1.00 & 0.75 & 1.00 & 1.00 & 0.65 & 1.00 & 0.00 & 0.88 \\
\hline $\begin{array}{c}\text { Konto } \\
\text { Optymalne- } \\
\text { PNB Paribas }\end{array}$ & 1.00 & 1.00 & 1.00 & 0.88 & 0.80 & 1.00 & 1.00 & 0.00 \\
\hline Millenium & 1.00 & 0.75 & 1.00 & 0.55 & 0.47 & 0.50 & 0.00 & 0.68 \\
\hline $\begin{array}{c}\text { Konto dla } \\
\text { Ciebie - } \\
\text { Credit } \\
\text { Agricole } \\
\end{array}$ & 1.00 & 1.00 & 1.00 & 0.55 & 0.80 & 1.00 & 1.00 & 0.00 \\
\hline $\begin{array}{c}\text { Konto Na } \\
\text { Zero - PKO } \\
\text { BP } \\
\end{array}$ & 1.00 & 0.83 & 0.44 & 0.71 & 0.70 & 0.75 & 0.00 & 0.00 \\
\hline $\begin{array}{c}\text { Konto Jakże } \\
\text { Osobiste - } \\
\text { Alior Bank }\end{array}$ & 1.00 & 0.75 & 0.33 & 0.94 & 0.90 & 1.00 & 0.00 & 0.00 \\
\hline $\begin{array}{c}\text { Konto } \\
\text { Freemium - } \\
\text { T-mobile } \\
\text { Usługi } \\
\text { Bankowe }\end{array}$ & 1.00 & 1.00 & 0.58 & 0.69 & 0.48 & 0.75 & 0.00 & 0.00 \\
\hline
\end{tabular}


Cont. table 9.

\begin{tabular}{|c|c|c|c|c|c|c|c|c|}
$\begin{array}{c}\text { Konto Jakie } \\
\text { Chcę- } \\
\text { Santander } \\
\text { Bank Polska }\end{array}$ & 1.00 & 1.00 & 0.50 & 0.71 & 0.72 & 1.00 & 0.00 & 0.00 \\
$\begin{array}{c}\text { Konto Bez } \\
\text { Granic-PKO } \\
\text { BP }\end{array}$ & 0.00 & 1.00 & 1.00 & 1.00 & 1.00 & 0.75 & 0.00 & 0.00 \\
\hline
\end{tabular}

Note: own study.

Taking into account the values of Level 1 criteria weights, total bank account assessments were obtained (results in Table 10). After normalising these ratings, there is a ranking presented according to the rating maximisation.

Table 10.

Summary of assessments and account ranking presentation

\begin{tabular}{|c|c|c|c|}
\hline & $\begin{array}{c}\text { Weighted assessments } \\
\text { according to the global } \\
\text { criterion }\end{array}$ & $\begin{array}{c}\text { Normalised weighted } \\
\text { assessment of universal } \\
\text { criterion }\end{array}$ & $\begin{array}{c}\text { Sorting according to } \\
\text { the optimal choice }\end{array}$ \\
\hline $\begin{array}{c}\text { Konto Direct - ING } \\
\text { Bank Sląsk }\end{array}$ & 0.775 & 0.812 & 1 \\
\hline $\begin{array}{c}\text { eKonto standard - } \\
\text { mBank }\end{array}$ & 0.788 & 0.826 & 0.977 \\
\hline $\begin{array}{c}\text { Nest Account - Nest } \\
\text { Bank }\end{array}$ & 0.757 & 0.793 & 0.883 \\
\hline $\begin{array}{c}\text { Konto Proste Zasady- } \\
\text { Getin Bank }\end{array}$ & 0.771 & 0.808 & 0.872 \\
\hline $\begin{array}{c}\text { Konto Optymalne }- \\
\text { PNB Paribas }\end{array}$ & 0.954 & 1.000 & 0.831 \\
\hline Millenium \\
\hline $\begin{array}{c}\text { Konto Dla Ciebie - } \\
\text { Credit Agricole }\end{array}$ & 0.609 & 0.639 & 0.807 \\
\hline $\begin{array}{c}\text { Konto Na Zero - PKO } \\
\text { BP }\end{array}$ & 0.907 & 0.950 & 0.765 \\
\hline $\begin{array}{c}\text { Konto Jakże Osobiste } \\
\text { - Alior Bank }\end{array}$ & 0.632 & 0.663 & 0.667 \\
\hline $\begin{array}{c}\text { Konto Freemium - } \\
\text { T-mobile Usługi } \\
\text { Bankowe }\end{array}$ & 0.703 & 0.737 & 0.665 \\
\hline $\begin{array}{c}\text { Konto Jakie Chce- }- \\
\text { Santander Bank Polska }\end{array}$ & 0.643 & 0.673 & 0.632 \\
\hline $\begin{array}{c}\text { Konto Bez Granic - } \\
\text { PKO BP }\end{array}$ & 0.704 & 0.738 & 0.602 \\
\hline
\end{tabular}

Note: own study.

Based on the proposed model, one can see which accounts (assuming the same value of criteria weights) are the most optimal. The highest rating received Konto Direct - ING Bank Śląski, eKonto standard - mBank also obtained a very high score, taking the 2nd position in the ranking. Konto Bez Granic received the lowest assessment - PKO BP. When analysing and interpreting the study it should be remembered that the result of the ranking could differ from that achieved when introducing changes in the weight values, which would better take into account the preferences of potential customers. 


\section{Summary}

Nowadays, the development of the banking market is extensive. Competition makes banks outdo each other in gaining new customers. The more possibilities, the more pros or cons on the side of one or the other bank, the bigger the uncertainty about choosing a bank account. Depending on the age, the amount of money invested, the frequency of trips abroad, expectations concerning increasing one's banking finances, etc., the choice of the variant will be different. The paper proposes a multicriterial model enabling comparison of accounts taking into account different values of individual criteria. For the example of calculations, the same weights were assumed, not guided by any subjective preferences. This variant assumes that all criteria have the same impact on the customer's decision. Based on such assumptions, the ranking of bank accounts was determined. Of course, the selection of the optimal bank account can be expanded by including analysis of other methods used for multiple purposes.

\section{References}

1. Brett, K. (2013). Bank 3.0. Nowy wymiar bankowości. Warszawa: Wydawnictwo studio Emka.

2. Kesra, N. (2017). Metody analizy wielokryterialnej i wielowymiarowej w podejmowaniu decyzji. Warszawa: PWN.

3. Miklaszewska, E. (ed.). Banki w nowym otoczeniu społecznym, gospodarczym i ekonomicznym. Warszawa: Wydawnictwo Poltext.

4. Stadnicki, J. (2006). Teoria i praktyka rozwiazywania zadań optymalizacyjnych. Warszawa: WNT.

5. Świderska, J., Klimontowicz, M., Galbarczyk, T. (2016). Banki komercyjne w Polsce. Warszawa: Difin.

6. Trzaskalik, T. (2006). Metody wielokryterialne na polskim rynku finansowym. Warszawa: PWE. 